\title{
Complementary and Alternative Medicine Use and Quality of Life among Filipino Adult Psoriasis Vulgaris Patients Seen in a Tertiary Center: A Cross-sectional Study
}

\author{
Marie Len A. Camaclang-Balmores, MD, ${ }^{1}$ Ma. Lorna F. Frez, MD, ${ }^{2}$ \\ Patricia A. Nacianceno, $\mathrm{MD}^{1}$ and Jay-V James G. Barit, $\mathrm{MD}^{1}$ \\ ${ }^{1}$ Department of Dermatology, Philippine General Hospital, University of the Philippines Manila \\ ${ }^{2}$ Department of Dermatology, College of Medicine and Philippine General Hospital, University of the Philippines Manila
}

\begin{abstract}
Objective. To determine the prevalence of complementary and alternative medicine (CAM) use and its association with quality of life (QOL) among Filipino adult psoriasis vulgaris patients.

Methods. A cross-sectional study was conducted in an outpatient dermatology department of a tertiary hospital, using a semi-structured, interview-guided questionnaire, and a self-administered QOL questionnaire, the dermatology life quality index (DLQI).
\end{abstract}

Results. A total of 135 Filipino adult patients with psoriasis vulgaris were included. The prevalence of CAM use was $47 \%$, with most CAM users being female and single. Completion of tertiary education was found significantly associated with CAM use $(p<0.05)$. A greater body surface area involvement and longer disease duration were more common among CAM users but these were not statistically significant. Special diet (56.3\%) was the most commonly used type of CAM, followed by herbal medicine (46.9\%), bath therapy (18.9\%) and faith healing (12.5\%). Major sources of CAM information were families (43.8\%), internet/social media (28.1\%) and health professionals (25\%). Around $40 \%$ of the participants used CAM out of curiosity. The mean DLQI score of the respondents was 11.3 $( \pm 7.3)$ corresponding to poor quality of life. CAM use was significantly associated with negative impact on physical symptoms and feelings, daily activities, and work and school $(P=0.044 ; P=0.019 ; P=0.047)$. After adjusting for confounding variables, patients with poor QOL were twice more likely to use CAM but this was not statistically significant (odds ratio [OR], 1.76; 95\% confidence interval [Cl], 0.78-3.95; $\mathrm{P}=0.17$ ).

Conclusions. The use of CAM is prevalent among Filipino adult patients with psoriasis vulgaris. The significant association between CAM use and a poor quality of life may reflect the unmet physical and psychosocial needs of patients. A patient-perspective approach should acknowledge the reasons for CAM use, which could guide the physicians in imparting available scientific evidence, or the lack thereof, for the use of CAM to these patients.

Key Words: alternative medicine, complementary medicine, Filipinos, psoriasis, quality of life

\section{INTRODUCTION}

Corresponding author: Marie Len A. Camaclang-Balmores, MD Department of Dermatology Philippine General Hospital University of the Philippines Manila

Taft Avenue, Manila 1000, Philippines

Email: mcbalmores1@up.edu.ph
Psoriasis is a chronic, inflammatory and immunemediated disease affecting $2 \%$ of the general population. ${ }^{1}$ Despite advances in management, the stigmatic lesions of psoriasis, amplified by its chronic nature, pose a substantial toll on the patients' quality of life (QOL). Patients face a lifelong ordeal of coping with the psychological and social impact of disease. Standard therapies for psoriasis do not offer definitive cure, as these treatments mainly control 
the disease, resulting in temporary remission of physical symptoms. These treatments are not devoid of long-term side effects and some, if not all, are costly and out-of-pocket. These biological and economic reasons preclude continuous and long-term treatment, and drive patients to seek other forms of therapies, either as adjunct or as substitute to conventional medicine. ${ }^{2}$

Complementary and alternative medicine (CAM) is defined as "a group of diverse medical and health care systems, practices and products that are not currently considered as part of conventional medicine." ${ }^{3}$ According to a systematic review in 2012, the lifetime prevalence of CAM usage ranged from $9.8-76 \%$ among the general population; ${ }^{4}$ and $35-69 \%$ in patients with skin diseases, ${ }^{5}$ especially among chronic and intractable diseases, such as atopic dermatitis, alopecia and psoriasis. ${ }^{6}$ Among patients with psoriasis, the prevalence on the use of CAM vary from $42-69 \% .^{7}$ In a study measuring the knowledge and attitudes on CAM use among dermatologists, $88.3 \%$ reported that patients asked information on CAM, and the most commonly inquired dermatologic disease was psoriasis (56.6\% of cases). ${ }^{8}$ In a local study among Philippine-based dermatologists, 66.67\% of patients who inquired about CAM were psoriasis patients. ${ }^{9}$ One reason for CAM use was seeking health improvement and better quality of life. ${ }^{10}$

The World Health Organization (WHO) defines quality of life as "a perception of life, perceived values, and interests in the scaffold of nature." Measurement of QOL has become increasingly relevant as health practitioners have shifted from the physician's standpoint to patient's perspective on seeking health outcomes. Despite a number of studies on the overall prevalence of CAM use in psoriasis patients, there are no existing published local studies on the subject. Furthermore, there are no published articles on the association of CAM use with quality of life among psoriasis patients.

The high prevalence of general CAM use in selected communities in the Philippines (51.1-68.4\%) ${ }^{11}$ and the growing and evolving body of research on the use of CAM among psoriasis patients across the world, highlight the need for local, data. With the ubiquity of CAM and it being considered non-evidence-based practice, while being recognized as an integral part in medical research, dermatologists must have an increased education on CAM. ${ }^{12}$ There is, however, foremost a crucial need to determine the current status of CAM use among psoriasis vulgaris patients.

Baseline local data on CAM use among psoriasis patients will guide the formulation of recommendations. Physicians, especially dermatologists, may use these data to provide more informed clinical care, which in turn would guide their patients' decision-making in terms of risks and benefits. Furthermore, this would guide future health-related quality of life research on CAM and ultimately educate both dermatologists and patients on evidence-based CAM use.

\section{MATERIALS AND METHODS}

\section{Setting and population}

This study was conducted at the outpatient department (OPD) of the Section of Dermatology, Philippine General Hospital (PGH), Manila, from July to October 2018. We recruited patients diagnosed with plaque-type psoriasis through consecutive sampling.

Inclusion criteria included Filipino adult patients aged nineteen years old and above who were clinically diagnosed with plaque-type psoriasis vulgaris at the outpatient department of the Section of Dermatology, Philippine General Hospital.

Exclusion criteria included patients who were unable to read and/or write, who had concomitant chronic skin disease other than psoriasis (e.g., atopic dermatitis) and who were hemodynamically unstable (e.g., hypotension, shortness of breath) needing immediate medical attention at the time of the study.

\section{Sample Size}

A total of 135 patients was required to estimate the prevalence of CAM use, assuming that the prevalence of CAM among this population is within $55 \%$ (with a margin of error of $8 \%$ ) at $95 \%$ confidence interval. This prevalence of CAM use is based on the study by Talbott and Duffy in 2015 wherein the prevalence estimations on the use of CAM among psoriasis patients were reported to vary from $42 \%$ to $69 \%{ }^{7}$

\section{Study procedure}

We administered the following in order: 1) consent form 2) interview-administered questionnaire 3) self-administered QOL questionnaire.

\section{Interview-administered questionnaire}

A face-to-face, interview-administered, semi-structured questionnaire was employed, which entailed an estimated time of ten (10) to twenty (20) minutes for each participant.

The interview-administered questionnaire was comprised of two parts. The first part consisted of questions on socio-demographic (sex, age, civil status, place of residence, educational level, employment and household income) and clinical characteristics (body surface involvement, disease duration, presence of a family history of psoriasis, current treatment, and presence of psoriatic arthritis). The second part opened with a screening question on CAM use. Participants who were identified as CAM users proceeded to answer questions on CAM-related characteristics. For this second part, guide cards with item-specific choices were utilized and participants were allowed to freely give answers to questions as deemed applicable. Prior to data collection, the interview-administered questionnaire underwent pre-testing and revisions before its administration to actual participants. 


\section{Self-administered QOL questionnaire}

The Dermatology Life Quality Index (DLQI) is a universally validated instrument for measuring healthrelated quality of life for skin diseases, ${ }^{13}$ with a validated Filipino version. It is a self-administered, dermatologyspecific questionnaire that has categorized six major health domains namely symptoms and feelings, daily activities, leisure, work or school, personal relationships and treatment. It consists of 10 multiple-choice items with a maximum total score of 30 and a minimum score of 0 .

\section{Outcome Variables}

\section{CAM use and CAM-related characteristics}

CAM use was defined as the use of CAM by participants at the time of the study or at least once in the last six months.

Among participants who used CAM, the type of CAM used, source of CAM information, perceived efficacy, disclosure to physician, recommendation and reasons for CAM use were collected.

\section{Quality of life}

The scoring system used in calculating the different domains and total scores was in accordance to the scoring algorithm of the DLQI questionnaire as originally outlined in the study by Finlay and Khan. ${ }^{13}$ The higher the score, the more impaired the quality of life. In this study, a score of 11-30 in the DLQI translated to a poor quality of life while a score of 10 or below translated to a good quality of life.

\section{Data Analysis}

Data analysis was performed using Stata SE version 13. Quantitative variables were summarized as mean and standard deviation, while qualitative variables were tabulated as frequency and percent. Baseline characteristics between CAM users and non-CAM users were compared using independent t-test for quantitative variables and Fisher's exact test for qualitative variables. Association of CAM use with quality of life was analyzed using logistic regression analysis. The level of significance was set at 5\%. Screening for confounders was done by performing simple logistic regression. All variables with $\mathrm{p}$-value $<0.25$ were considered as possible confounders and were later on tested using percent change in estimate criterion. A 10\% change in estimate suggested that the confounder being investigated should be included in the model which was determined using multiple logistic regression.

\section{Ethical Considerations}

The research protocol was approved by the University of the Philippines Manila Research Ethics Board (UPMREB). Informed consent forms, containing the objectives of the study, conditions for voluntary withdrawal and confidentiality clauses, were obtained prior to inclusion. The investigators ensured that all personal identifiers would be kept confidential. All data collection forms were stored in a secure location during the duration of the study.

\section{RESULTS}

A total of 135 participants were included in the study. The sociodemographic and clinical profiles are presented in Table 1. The mean age of participants was 42.1 years (range, 19 to 85 ). Majority of the participants were females $(62.5 \%)$, unmarried $(57 \%)$, and unemployed (66\%), with a net family income of more than 10,000 Php (57\%). There were also more CAM users who completed tertiary education, which was found to be significantly associated ( $\mathrm{p}$ $<0.05$ ). For the clinical profile, majority of the respondents had less than $10 \%$ body surface involvement $(58.5 \%)$ and the mean disease duration was 7.9 years. There were more patients with greater than 30\% BSA and longer disease duration among the CAM users compared to non-CAM users but these were not statistically significant. More than thirty percent (32.6\%) had a family history of psoriasis, and only $14.8 \%$ had psoriatic arthritis. Most of the participants (77.8\%) had topical standard medications and 23\% had non-standard medications including oral antihistamines, lotions/emollients (e.g., petroleum jelly) and mineral oil as their current treatment.

Majority (75\% or 101/135) of patients used CAM at least once since their diagnosis, and 64 patients (47\%) used CAM in the previous six months. Special diet was the most commonly used CAM modality, followed by herbal medicine, bath therapy and faith healing (Table 2). Fortyone of CAM users (64.1\%) used only one type of CAM. On average, a CAM user utilizes 1.4 (range, 1 to 4) modalities. Details on the most commonly used multi-component modalities (special diet, herbal medicine, bath therapy) are presented in Table 3.

Majority of CAM users stated that they learned about CAM from families and relatives (43.8\%). The internet and social media were the source of CAM information in $28.1 \%$, and health professionals in $25 \%$.

About twenty seven percent of CAM users perceived no efficacy from CAM use, although eight patients (12.5\%) utilizing more than one type of CAM had mixed answers. Two patients who were on special diet, one on a vegetarian diet and another on mangosteen food supplement, reported CAM to be very effective. Majority of the respondents did not disclose CAM use to their physicians (54.7\%) and would not recommend CAM use to other psoriasis patients (46.9\%). A more detailed descriptive analysis based on the types of CAM revealed that most patients who used faith healing perceived no efficacy, while prayer/spiritual healing was perceived to be effective. In addition, most of the patients who used special diet and spiritual healing disclosed to their physicians and stated that they would recommend the CAM to fellow psoriasis patients. 
Table 1. Socio-demographic and clinical profile of CAM users and non-CAM users among adult Filipino patients with psoriasis vulgaris

\begin{tabular}{|c|c|c|c|}
\hline Variables & $\begin{array}{c}\text { CAM users } \\
(n=64)\end{array}$ & $\begin{array}{c}\text { CAM non-users } \\
(n=71)\end{array}$ & p-value \\
\hline \multicolumn{4}{|c|}{ Socio-demographic characteristics } \\
\hline \multicolumn{4}{|l|}{ Sex } \\
\hline Male & $23(35.9)$ & $29(40.9)$ & \multirow{2}{*}{0.56} \\
\hline Female & $41(64.1)$ & $42(59.2)$ & \\
\hline Age (mean, SD) (yrs.) & $40.8(15.7)$ & $43.2(17.7)$ & 0.42 \\
\hline \multicolumn{4}{|l|}{ Civil status } \\
\hline Single & $31(48.4)$ & $26(36.6)$ & \multirow{4}{*}{0.14} \\
\hline Married & $21(32.8)$ & $37(52.1)$ & \\
\hline Widow/Widower & $8(12.5)$ & $6(8.5)$ & \\
\hline Separated & $4(6.3)$ & $2(2.8)$ & \\
\hline \multicolumn{4}{|l|}{ Residence } \\
\hline Urban & $10(15.6)$ & $10(14.1)$ & \multirow{2}{*}{0.18} \\
\hline Rural & $54(84.4)$ & $61(85.9)$ & \\
\hline \multicolumn{4}{|l|}{ Educational level } \\
\hline Elementary & $1(1.6)$ & $10(14.1)$ & \multirow{4}{*}{$0.005^{*}$} \\
\hline High school & $25(39.7)$ & $32(45.1)$ & \\
\hline College & $33(52.4)$ & $29(40.9)$ & \\
\hline Vocational & $4(6.4)$ & $0(0.0)$ & \\
\hline \multicolumn{4}{|l|}{ Employment } \\
\hline Unemployed & $43(67.2)$ & $46(64.8)$ & \multirow{2}{*}{0.77} \\
\hline Employed & $21(32.8)$ & $25(35.2)$ & \\
\hline \multicolumn{4}{|l|}{ Income (Php) } \\
\hline$<5000$ & $5(7.8)$ & $13(18.3)$ & \multirow{3}{*}{$0.07^{*}$} \\
\hline $5000-10000$ & $24(37.5)$ & $16(22.5)$ & \\
\hline$>10000$ & $35(54.7)$ & $42(59.2)$ & \\
\hline \multicolumn{4}{|c|}{ Clinical characteristics } \\
\hline \multicolumn{4}{|c|}{ Body surface involvement (\%) } \\
\hline$<10 \%$ & $34(53.1)$ & $45(63.4)$ & \multirow{3}{*}{$0.08^{*}$} \\
\hline $10-30 \%$ & $12(18.8)$ & $17(24.0)$ & \\
\hline$>30 \%$ & $18(28.1)$ & $9(12.7)$ & \\
\hline \multicolumn{4}{|c|}{ Disease Duration (years) } \\
\hline$<1$ year & $18(28.1)$ & $16(22.5)$ & \multirow{3}{*}{0.51} \\
\hline $1-5$ years & $19(29.7)$ & $18(25.4)$ & \\
\hline$>5$ years & $27(42.2)$ & $37(52.1)$ & \\
\hline \multicolumn{4}{|l|}{ Family history } \\
\hline Yes & $21(32.8)$ & $23(32.4)$ & \multirow{2}{*}{0.96} \\
\hline No & $43(67.2)$ & $48(67.6)$ & \\
\hline \multicolumn{4}{|l|}{ Current medications $^{\dagger}$} \\
\hline Topical & $48(75)$ & $57(80.2)$ & 0.54 \\
\hline Systemic & $12(18.8)$ & $12(18.3)$ & 1 \\
\hline Phototherapy & $3(4.7)$ & $3(4.2)$ & 1 \\
\hline Biologics & $3(4.7)$ & $2(2.8)$ & 0.67 \\
\hline Others & $12(18.8)$ & $19(26.8)$ & 0.31 \\
\hline \multicolumn{4}{|c|}{ Presence of psoriatic arthritis } \\
\hline Yes & $10(15.6)$ & $10(14.1)$ & 0.81 \\
\hline No & $54(84.4)$ & $61(85.9)$ & \\
\hline Quality of life & & & \\
\hline Poor & $40(56.34)$ & $31(43.66)$ & $0.03^{*}$ \\
\hline Good & $24(37.5)$ & $40(62.5)$ & 0.03 \\
\hline
\end{tabular}

${ }^{*}$ Variables with $p$-value $<0.25$ were considered as possible confounders † Multiple answers
Table 2. CAM practices among adult Filipino psoriasis vulgaris patients

\section{CAM practices $(n=64)$}

Frequency, n (\%)

\section{Types of CAM*}

Special diet

Herbal medicine

$6(56.3)$

Bath therapy

$30(46.9)$

Faith healer

$11(18.9)$

Spiritual healing

8 (12.5)

Chinese medicine

5 (7.8)

Therapeutic massage

$2(3.1)$

Mind-body therapy

$1(1.6)$

$1(1.6)$

Energy medicine

$1(1.6)$

Sources of CAM*

From family/relative

$28(43.8)$

Internet/Social Media

$18(28.1)$

From health professional

$16(25)$

From peers

$12(18.8)$

Media (TV/radio)

8 (12.5)

Personal choice

8 (12.5)

From a patient

$4(6.3)$

Others

$3(4.7)$

Print ad (newspaper/magazine/books)

2 (3.1)

\section{Perceived efficacy}

Not effective

$17(26.6)$

Slightly effective

$22(34.4)$

Effective

$15(23.4)$

Very effective

2 (3.1)

Undecided $^{\dagger}$

$8(12.5)$

\section{Disclosure to physician}

None

$35(54.7)$

Full

$27(42.2)$

Partial

2 (3.1)

\section{Recommendation}

No $\quad 30(46.9)$

Yes 20 (31.3)

Undecided $^{\dagger} \quad 8$ (12.5)

Partial

$6(9.4)$

Reason for CAM use*

Curiosity

Cheaper than prescribed treatment

$26(40.6)$

Others

$22(34.4)$

Tradition or culture

$21(32.8)$

$19(29.7)$

Acquaintance with product or practice

14 (21.9)

Safer than prescribed treatment

$14(21.9)$

More accessible than prescribed treatment

7 (10.9)

More effective than prescribed treatmen

7 (10.9)

* Multiple answers; † Patients used more than one type of CAM

Most patients who used CAM did so out of curiosity (40.6\%). A third of patients (32.8\%) had other reasons such as recommended/influenced by sources, for general health/ immune system, as supplement to prescribed meds, and as alternatives to standard treatment.

\section{Quality of life}

There was a very large effect on the quality of life of respondents (mean DLQI score 11.3, SD 7.3, range 11 to 20). Overall, there was a statistically significant difference 
Table 3. List of CAM according to the most commonly used multicomponent CAM modalities

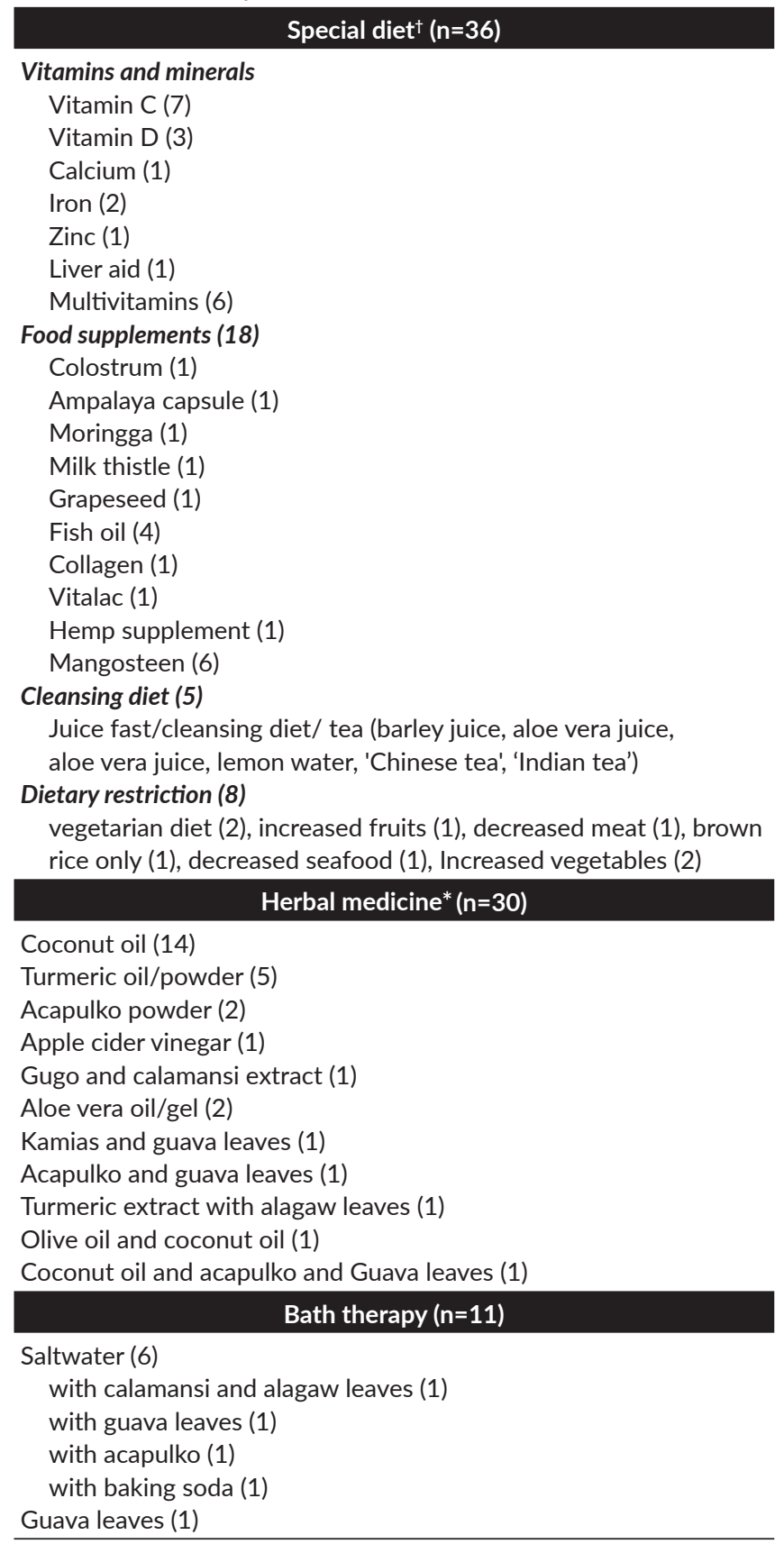

${ }^{*}$ All were used topically; $†$ Multiple answers

in mean DLQI scores between CAM user and non-CAM user groups $(\mathrm{p}=0.017)$. (Table 4).

Out of 64 respondents who were CAM users, 40 (56.3\%) had a total DLQI score of 11 to 30 , corresponding to a poor quality of life. Multiple logistic regression analysis, to account for confounding variables (variables with $\mathrm{p}$-value $<0.25$ ), showed that patients with poor QOL were 1.76 times more likely to use CAM than patients with good QOL (Table 5). However, this was not significant (95\% CI 0.78-3.95). The three health domains related to symptoms
Table 4. Comparison of total DLQI scores between CAMusers and non-CAM users among adult psoriasis vulgaris patients

\begin{tabular}{|c|c|c|c|}
\hline $\begin{array}{l}\text { Total DLQI } \\
\text { score }\end{array}$ & $\begin{array}{c}\text { CAM use }(n=64) \\
n(\%)\end{array}$ & $\begin{array}{c}\text { Non-CAM use }(n=71) \\
n(\%)\end{array}$ & p-value \\
\hline 0 to 1 & $1(1.6)$ & $11(15.5)$ & \multirow{5}{*}{0.017} \\
\hline 2 to 5 & 7 (10.9) & 14 (19.7) & \\
\hline 6 to 10 & $16(25.0)$ & $15(21.1)$ & \\
\hline 11 to 20 & $30(46.9)$ & $23(32.3)$ & \\
\hline 21 to 30 & 10 (15.6) & 8 (11.2) & \\
\hline
\end{tabular}

0-1: No effect at all on patient's life; 2-5: Small effect on patient's life; 6-10: Moderate effect on patient's life; 11-20 Very large effect on patient's life; 21-30: Extremely large effect on patient's life

Table 5. CAM use and QOL among adult psoriasis vulgaris patients

\begin{tabular}{ccccc} 
& $\begin{array}{c}\text { CAM use } \\
(\mathbf{n}=64) \\
\mathbf{n}(\%)\end{array}$ & $\begin{array}{c}\text { Non-CAM } \\
\text { use } \\
(\mathbf{n}=\mathbf{7 1}) \\
\mathbf{n}(\%)\end{array}$ & $\begin{array}{c}\text { Odds Ratio* } \\
(\mathbf{9 5 \%} \mathrm{Cl})\end{array}$ & p-value \\
\cline { 1 - 3 } Poor QOL & $40(56.3)$ & $31(43.7)$ & & \\
\hline Good QOL & $24(37.5)$ & $40(62.5)$ & & \\
\hline
\end{tabular}

* adjusted odds ratio

Table 6. Comparison of DLQI scores per health domain according to CAM use among adult psoriasis vulgaris patients

\begin{tabular}{lccc}
\multicolumn{1}{c}{ Health Domain } & $\begin{array}{c}\text { CAM use } \\
\text { Median DLQI }\end{array}$ & $\begin{array}{c}\text { Non-CAM use } \\
\text { Median DLQI }\end{array}$ & p-value \\
\hline Symptoms/feelings & 2 & 3 & 0.02 \\
Daily activities & 2 & 3 & 0.01 \\
Leisure & 2 & 2 & 0.07 \\
Work or school & 1 & 1 & 0.04 \\
Personal relationships & 1 & 1 & 0.15 \\
Treatment & 1 & 1 & 0.05 \\
\hline
\end{tabular}

and feelings, daily activities and work and school showed significant association with CAM use (95\% CI, 1.99-2.77, 1.68-2.60, 0.95-1.56). (Table 6).

\section{DISCUSSION}

This study shows a high prevalence of CAM use among adult patients with psoriasis vulgaris seen in the outpatient department, which is within the range of prevalence estimations at $42-69 \% .^{7}$ This prevalence estimate, however, must be taken with caution because of the non-standardized definition of CAM across these studies. Estimates may be influenced by cultural, social and methodological factors, and underreporting from non-disclosure of CAM use to physicians. Demographic data on CAM use among patients with psoriasis parallel the general and dermatologic CAM trends in literature in terms of sex, age and education. ${ }^{4,14-18}$ Similar to the findings in a national survey among the general population in the United States, ${ }^{17}$ majority of 
CAM users were females (64.1\%) in their fourth decade. who have completed tertiary level of education (52.4\%). This is comparable to previous studies that found increased likelihood of using CAM among those with a higher level of education; ${ }^{16,19,20}$ perhaps reflecting a more health-conscious subset of the population. Although most CAM users were unemployed, majority of these patients may be considered economically able, belonging to income-generating families.

Although there were more CAM users among patients with greater than 30\% BSA involvement and longer disease duration, these were not significant between the two groups. This is contrary to previous prevalence studies which found that the chronicity and severity of psoriasis were associated with CAM use; $;^{20,21}$ however, in another study, disease duration and severity were likewise not significant with CAM use, suggesting a need for further investigation. ${ }^{2}$ In addition, the presence of psoriatic arthritis failed to show a significant association with CAM use, despite nonspecific arthritis or joint stiffness being listed as one of the common reasons for CAM use in the general population..$^{22}$

Special diet was the most commonly used modality, similar with earlier reports on CAM., ${ }^{5,6,23}$ In a recent systematic review on CAM for the treatment of psoriasis, the authors concluded that dietary approaches have one of the most evidence supporting clinical use. Fish oil was included in this list, despite conflicting results of randomized controlled trials. ${ }^{24}$ Ranking second, herbal medicine and its prevalent use may be influenced by the rich, local tradition of using "balamang-gamot." The belief that these herbal products are 'natural' and 'harmless' may also contribute to their widespread use. ${ }^{7}$ Interestingly, a number of patients still sought consult with a faith healer/ albularyo (12.5\%), albeit most of these respondents stated lack of efficacy. Saltwater bath therapy was a common practice among patients with chronic skin conditions, namely, atopic dermatitis, androgenetic alopecia and psoriasis. ${ }^{6}$ The use of salt therapy may be influenced by clinical efficacy reported from bathing in the Dead Sea as well as artificial climatotherapy. ${ }^{7,25}$ Spiritual healing or prayer remains an integral part of the local culture among Christian nations. ${ }^{11}$

Majority of the patients used CAM based on the inputs from their families/relatives and from the social media. An earlier local study on general CAM use also showed that a majority of CAM users chose their CAM therapy based on their families' recommendations which emphasizes the closeknit relationship among Filipino families. ${ }^{11}$ Only $18 \%$ stated that the CAM was recommended by a health professional. In a cross-sectional survey among local dermatologists, $81.8 \%$ had a positive attitude with giving CAM as complement to standard treatment of skin conditions including psoriasis, and $82 \%$ recommended herbal medicine. ${ }^{9}$ The popularity of herbal medicine and special diets may reflect the increasing number of clinical trials on these modalities, and consequent recommendations by health professionals. ${ }^{24}$ Furthermore, the perceived lack of efficacy of CAM by most patients may suggest that more studies are needed to strengthen the available evidence. Perceived efficacy may also be influenced by the duration and frequency of use, adverse effects, and patients' expectations, which were beyond the scope of this study.

This study also showed that majority did not disclose CAM use to their physicians. This finding is similar across studies in a 2004 systematic review by Robinson and Grail, which outlined fear of negative feedback from their physician, perception that physician is ignorant of $\mathrm{CAM}$, and physicians not raising the issue, as reasons for non-disclosure. ${ }^{26}$ In this study, some participants voluntarily offered reason for non-disclosure, including the reason that the information was not asked by their physician.

Similar to a local prevalence study on CAM, curiosity and affordability were the main reasons for CAM use. ${ }^{11}$ Thirty-four percent also reported that CAM was cheaper than their current standard treatment, while only $10.9 \%$ stated that CAM was more effective than prescribed treatment. This may reflect the use of CAM by patients looking for costeffective alternatives, and not necessarily because of lack of success with standard treatment. More than thirty percent (34.4\%) of patients answered that they were also after the overall benefit of using CAM for psoriasis, indicating that CAM boosted one's general health and immune system. This is comparable to a previous study which reported that the main reason for CAM use was 'doing everything to heal the disease.' ${ }^{2}$ This may suggest the desire of patients to find "complementary" rather than "alternative" treatment for psoriasis. However, it is also noteworthy that a number of patients indicated that they used CAM because these were recommended by others and that they were looking for alternative treatment. This may represent a subset of patients that have not been well-informed regarding the chronicity of psoriasis or the absence to date of a definitive cure.

The negative impact of psoriasis on QOL, as denoted by high DLQI scores in this study, have long been established in previous studies. ${ }^{27,28}$ Among CAM users, a low QOL was previously reported, but the population consisted of cancer patients. ${ }^{10,23,29}$ Nevertheless, the negative impact of psoriasis on QOL was found comparable with similar chronic conditions. ${ }^{30}$ Accounting for body surface area involvement, household income and educational attainment, the odds of CAM use among patients with poor QOL was lower. This showed that the outcome may not be attributed to having poor QOL alone.

Taking into account the significant association of CAM use with domains related to emotional, social and occupational functions, the impairment caused by psoriasis in these domains may drive them to use CAM. These domains involve a patient's self-esteem and interaction with other people; hence the association with CAM use may reflect the patient's desire to address an impaired selfimage. Interestingly, only $10.9 \%$ reported CAM as more effective than standard treatment, supporting a perceived 
lack of efficacy of standard treatment. This may explain the high number of patients with poor QOL among CAM users. There was significant effect on health domains related to symptoms and feelings, daily activities, and work and school. This observed association may reflect patients' desire to address the negative physical and psychosocial impact of psoriasis not adequately managed by standard therapy. Likewise, the unmet needs not addressed by CAM use may also lead to a poor quality of life. Addressing the quality of life of psoriasis patients should not be a one-way feat. Hence, the non-disclosure of CAM use to physicians raises concern on patient-doctor communication. Considering the high number of CAM users among psoriasis patients, the dermatologist should keep an open mind on patients possibly seeking for other forms of treatment, either as an adjunct or an alternative. Adapting a patient-perspective approach should not however, tolerate the use of CAM without informed clinical care; rather, it should acknowledge the patients' reasons for CAM use. While there are still gaps in research that need to be addressed with regards to CAM use, it is the physician's role to emphasize the current available evidence, or the lack thereof, of CAM to their patients, in the management of chronic skin conditions such as psoriasis.

\section{Limitations}

This study acknowledges the broad, often overlapping, scope of CAM, and the possible cultural differences that may influence the choice of CAM. For this reason, the CAM modalities presented as primary options in the interviewguided questionnaire were categories based on the prevalence of CAM modalities reported in the literature review with focus on Asian and local studies. ${ }^{6,7,9,11,31}$ There is also currently no validated tool in assessing CAM use.

Another limitation of the study is the absence of delineation between 'complementary' and 'alternative' medicine which might have contributed with the ambiguity in the perceived efficacy of CAM. The study also did not explore the specific benefits and possible adverse effects, the duration and frequency of CAM use, as well as patients' expectations related to CAM use, which might be influential to the perceived efficacy.

The participants in this study were limited to patients with psoriasis seen at the outpatient department; hence, data obtained does not reflect the general population. The setting might have also triggered a social desirability bias and might have influenced patient's response on CAM practices, including their willingness to "admit" non-disclosure. Surprisingly, a high number of participants admitted nondisclosure to physicians despite the inquiry taking place in a dermatology department. However, reasons for nondisclosure were not explored. The design is also susceptible to recall bias as respondents may not fully remember history of CAM use and practices.

Lastly, a cross-sectional study design could not establish the directionality of the association between CAM use and
QOL. Hence, the cause-effect relationship cannot be fully established: it could not be determined if the poor quality of life drives patients to use CAM, or vice-versa, i.e., the use of CAM leads patients to have poor quality of life.

\section{CONCLUSION}

CAM use is prevalent among Filipino adult patients with psoriasis, more commonly among single, females in their fourth decade, who have completed tertiary education, patients with greater body surface area involvement and longer disease duration. Special diet, herbal medicine, bath therapy and faith healing were the most commonly used CAM modalities. Poor quality of life was associated with CAM use, particularly in the health domains of symptoms and feelings, daily activities and work and school.

Local research focusing on the efficacy and safety of specific components of CAM modalities in the treatment of psoriasis would be useful for future clinical recommendations. A qualitative research method may also provide a more indepth analysis of outcome variables, specifically of CAM practices, in association with perceived quality of life. Discussions on CAM, which are not routinely integrated in medical training, in continuing medical education (CME) sessions, would also keep the dermatologists updated and competent in providing reliable CAM information to their patients.

\section{Statement of Authorship}

All authors participated in data collection and analysis, and approved the final version submitted.

\section{Author Disclosure}

All authors declared no conflicts of interest.

\section{Funding Source}

This paper was self-funded.

\section{REFERENCES}

1. Gelfand JM, Weinstein R, Porter SB, Neimann AL, Berlin JA, Margolis DJ. Prevalence and treatment of psoriasis in the United Kingdom: a population-based study. Arch Dermatol. 2005; 141(12):1537-41.

2. Ben-Arye E, Ziv M, Frenkel M, Lavi I, Rosenman D. Complementary medicine and psoriasis: linking the patient's outlook with evidencebased medicine. Dermatology. 2003;207(3):302-7.

3. Passarelli T. Complementary and alternative medicine in the United States [Internet]. 2008. [cited 2018 March 10]. Available from: https://studylib.net/doc/10284871/complementary-and-alternativemedicine-in-the-united-stat...

4. Harris PE, Cooper KL, Relton C, Thomas KJ. Prevalence of complementary and alternative medicine (CAM) use by the general population: a systematic review and update: CAM use by general population. Int J Clin Pract.. 2012; 66(10):924-39.

5. Ernst E. The usage of complementary therapies by dermatological patients: a systematic review: Br J Dermatol. 2000; 142(5):857-61.

6. Kim G-W, Park J-M, Chin H-W, Ko H-C, Kim M-B, Kim J-Y, et al. Comparative analysis of the use of complementary and alternative medicine by Korean patients with androgenetic alopecia, atopic 
dermatitis and psoriasis. J Eur Acad Dermatol Venereol. 2013; 27(7):827-35.

7. Talbott W, Duffy N. Complementary and alternative medicine for psoriasis: what the dermatologist needs to know. Am J Clin Dermatol. 2015; 16(3):147-65.

8. Renzi C, Mastroeni S, Paradisi M, Mazzotti E, Pasquini P. Complementary and alternative medicine: knowledge and attitudes among dermatologists. Acta Derm Venereol. 2009; 89(6):642-4.

9. Pardo IA, Maano C. Complementary and alternative medicine for skin diseases: knowledge, attitudes and practices of Philippine-based dermatologists. J Phil Dermatol Soc. 2013; 22(2):26-35.

10. Erku DA. Complementary and alternative medicine use and its association with quality of life among cancer patients receiving chemotherapy in Ethiopia: a cross-sectional study. Evid Based Complement Alternat Med. 2016; Article ID 2809875, 8 pages.

11. Dahilig VRA, Salenga RL. Prevalence, perceptions and predictors of complementary and alternative medicine use in selected communities in the Philippines. Journal of Asian Association of Schools of Pharmacy. 2012; 2(1):16-24.

12. Bhuchar S, Katta R, Wolf J. Complementary and alternative medicine in dermatology: an overview of selected modalities for the practicing dermatologist. Am J Clin Dermatol. 2012; 13(5):311-7.

13. Finlay AY, Khan GK. Dermatology Life Quality Index (DLQI) - a simple practical measure for routine clinical use. Clin Exp Dermatol. 1994; 19(3):210-6.

14. Baron SE, Goodwin RG, Nicolau N, Blackford S, Goulden V. Use of complementary medicine among outpatients with dermatologic conditions within Yorkshire and South Wales, United Kingdom. J Am Acad Dermatol. 2005; 52(4):589-94.

15. Chen Y-F, Chang JS. Complementary and alternative medicine use among patients attending a hospital dermatology clinic in Taiwan. Int J Dermat. 2003; 42(8):616-21.

16. Fuhrmann T, Smith N, Tausk F. Use of complementary and alternative medicine among adults with skin disease: Updated results from a national survey. J Am Acad Dermatol. 2010;-63(6):1000-5.

17. Landis ET, Davis SA, Feldman SR, Taylor S. Complementary and alternative medicine use in dermatology in the United States. J Altern Complement Med 2014; 20(5):392-8.

18. Smith N, Shin DB, Brauer JA, Mao J, Gelfand JM. Use of complementary and alternative medicine among adults with skin disease: results from a national survey. J Am Acad Dermatol. 2009; 60(3):419-25.
19. Dastgheib L, Farahangiz S, Adelpour Z, Salehi A. The prevalence of complementary and alternative medicine use among dermatology outpatients in Shiraz, Iran. Evid Based Complement Alternat Med. 2017; 22(4):731-5.

20. See A, Teo B, Kwan R, Lim R, Lee J, Tang MB, et al. Use of complementary and alternative medicine among dermatology outpatients in Singapore: Complementary and alternative medicine. Australas J Dermatol 2011; 52(1):7-13.

21. Ernst E. CAM in dermatology: Telling fact from fiction. Int $J$ Dermatol. 2003; 42(12):979-80.

22. Barnes PM, Bloom B, Nahin RL. Complementary and alternative medicine use among adults and children: United States, 2007. National Health Statistics Report. 2008; (12):1-4.

23. Naja F, Fadel RA, Alameddine M, Aridi Y, Zarif A, Hariri D, et al. Complementary and alternative medicine use and its association with quality of life among Lebanese breast cancer patients: a crosssectional study. BMC Complementary and Alternative Medicine. 2015; 15(1):444.

24. Gamret AC, Price A, Fertig RM, Lev-Tov H, Nichols AJ. Complementary and alternative medicine therapies for psoriasis: a systematic review. JAMA Dermatology. 2018;E1-9.

25. Kopel E, Levi A, Harari M, Ruzicka T, Ingber A. Effect of the dead sea climatotherapy for psoriasis on quality of life. Isr Med Assoc J. 2013; 15(2):99-102.

26. Robinson A, McGrail MR. Disclosure of CAM use to medical practitioners: a review of qualitative and quantitative studies. Complement Ther Med 2004; 12(2-3):90-8.

27. Bhosle MJ, Kulkarni A, Feldman SR, Balkrishnan R. Quality of life in patients with psoriasis. Health Qual Life Outcomes. 2006; 4(35).

28. Khoury LR, Danielsen PL, Skiveren J. Body image altered by psoriasis. A study based on individual interviews and a model for body image. J Dermatolog Treat 2014; 25(1):2-7.

29. Korkmaz M, Tavsanli NG, Ozcelik H. Use of complementary and alternative medicine and quality of life of cancer patients: Turkish Samples. Holist Nurs Pract. 2016; 30(2):88-95.

30. Rapp SR, Feldman SR, Exum ML, Fleischer AB, Reboussin DM, Carolina N. Psoriasis causes as much disability as other major medical diseases. J Am Acad Dermatol. 1999; 41(3):401-7.

31. Damevska K, Neloska L, Nikolovska S, Gocev G, Duma S. Complementary and alternative medicine use among patients with psoriasis: complementary medicine and psoriasis. Dermatol Ther. 2014; 27(5):281-3. 